\title{
Noninvasive radiation burn diagnosis using speckle phenomenon with a fractal approach to processing
}

\author{
Odile Carvalho \\ Marc Benderitter \\ Laurence Roy \\ Institut de Radioprotection et de Sûreté Nucléaire \\ Direction de la Radioprotection de l'Homme \\ Service de Radiobiologie et d'Epidémiologie \\ Fontenay-aux-Roses Cedex, 92262 France
}

\begin{abstract}
Radiation burns account for the vast majority of damage by accidental radiation exposure. They are characterized by successive and unpredictable inflammatory bursts that are preceded by a clinically latent postirradiation period. Diagnosis and prognosis of the clinical course of radiation burns have proven to be a difficult task. In a classical clinical setting, no technique can distinguish irradiated versus healthy skin during the clinically latent period, hence development of new tools is required. This work describes a noninvasive technique based on speckle phenomenon, designed to support radiation burn diagnosis and prognosis. Speckle produced by strongly scattering media contains information about their optical properties. The difficulty is to extract significant information from speckle patterns to discriminate between strongly scattering media and to characterize any change. Speckle patterns from irradiated and nonirradiated porcine skins are recorded in vivo several times after radiation exposure. A fractal approach is used in the treatment of speckle patterns. The results show that this technique allows discrimination between healthy and irradiated skin, in particular during the clinically latent period $(p<0.01)$. Parameters extracted from speckle patterns discriminate and vary differently with radiation, which means they represent different information about skin changes. () 2010 Society of PhotoOptical Instrumentation Engineers. [DOI: 10.1117/1.3394261]
\end{abstract}

Keywords: radiation burns; skin; diagnosis; speckle; fractal; fractional Brownian motion.

Paper 09217RR received Jun. 3, 2009; revised manuscript received Feb. 26, 2010; accepted for publication Mar. 1, 2010; published online Apr. 16, 2010.

\section{Introduction}

Increase in the number of radioactivity applications throughout the twentieth century inevitably resulted in an increase in radiological accidents in the industrial and medical sectors. Radiation exposure can be differentiated in localized and whole-body exposures. Localized radiation exposures are the most frequent. The skin is the first organ exposed during radiation exposure. Specific characteristics of radiation burns, compared with thermal burns, are the postirradiation delay in the onset of clinical changes and the successive and unpredictable course of inflammatory bursts. The delay before emergence of clinical symptoms is notably inversely proportional to the dose received. In many cases, patients are only aware of the radiation injury when first symptoms appear, usually several weeks after radiation exposure. The severity of radiation injuries mainly depends on the radiation source, dose and dose rate, exposure duration, dose distribution within the irradiated tissue in surface and depth, and on the body location and size of the area exposed. ${ }^{1}$ Due to their special features, medical handling of radiation burns requires very specialized care with extremely time- and cost-intensive

Address all correspondence to: Odile Carvalho, Institut de Radioprotection et de Sûreté Nucléaire, Direction de la Radioprotection de l'Homme, Service de Radiobiologie et d'Epidémiologie, Fontenay-aux-Roses Cedex, 92262 France. Tel: +331-58-35-82-06; Fax: +331-58-35-84-67; E-mail: odile.carvalho@irsn.fr medical treatment. Early and accurate diagnosis is essential to increase treatment efficacy. Diagnostic efficiency is based on determination of the extent of damage, and on a good dosimetry assessment of the area exposed in surface and depth, as precisely and as early as possible.

The dosimetric evaluation of radiation burns is usually based on the progression of visible clinical signs and on the physical reconstruction of the dose distribution by numerical means based on Monte Carlo simulations. However, it is problematic for clinicians to refer to clinical signs because of the clinically latent postirradiation phase. Thus, the diagnosis may be delayed after radiation exposure and the treatment compromised. Moreover, one of the major difficulties of the dose physical reconstruction, which is based on accident witness reports, is the lack of accurate knowledge of the accident scenario such as exposure type, distance from the radioactive source, and exposure duration. Hence, in an accident context, the diagnosis from physical reconstruction is often imprecise and the therapeutic success uncertain. Dose physical reconstruction associated with ex vivo electron paramagnetic resonance (EPR) measurements have been used in dosimetric evaluation in an accident context. ${ }^{2}$ However, EPR measurements are invasive, owing to the need to extract bone samples from patients for localized dose evaluation. ${ }^{2,3}$ EPR dosimetry

1083-3668/2010/15(2)/027013/13/\$25.00 @ 2010 SPIE 
in fingernails is a promising noninvasive tool, but can only provide dose measurement in hands or feet. ${ }^{4}$ Biodosimetric techniques based on scoring of chromosomal aberrations in fibroblasts have proven useful for localized dose evaluation. However, these methods are invasive, time consuming, and seemingly unreliable for high doses $(>10 \mathrm{~Gy}) .^{5}$

To improve the care of patients overexposed to ionizing radiation, it is important to develop tools that improve the diagnosis and prognosis of radio-induced lesions. Various physical techniques can help diagnosis after radiation overexposure, even though they cannot be considered as indicators of dose. Among the most relevant, clinical tools used for thermal burn diagnosis, such as a scanner, laser Doppler, MRI, ${ }^{6,7}$ and ultrasound techniques, ${ }^{8-10}$ have been tested to distinguish irradiated from healthy skin. Clinically and histologically, radiation burns show similarities to thermal burns. However, diagnostic tools used in the case of thermal burns are not efficient for radiation burns when there is no clinical sign. ${ }^{11-15}$ Therefore, these tools are inefficient for early diagnosis of radio-induced lesions. Feedback from clinicians shows that currently there is no tool able to efficiently detect radiation damage when it is not visible.

A new technique is proposed to discriminate in vivo radiation injury, in particular when the clinician is unable to delimit the irradiated zone. This technique uses the speckle phenomenon. Speckle can be considered noise in imaging, but it is a phenomenon that arises directly from the interaction between coherent light and medium. Therefore, speckle statistical properties are related to medium properties. Several speckle applications have been developed in stellar physics, many in industry for the study of roughness, deformation, displacement, or heterogenity of objects. ${ }^{16-20}$ In the biomedical field, measurement of several spatial or dynamic characteristics of speckle can give useful information for medical diagnosis. Thus, correlation methods ${ }^{21,22}$ or laser speckle contrast measurements ${ }^{23,24}$ can be used to analyze the motion of speckle field from blood flows. Several authors have studied bone deformation or bone implant deformation by speckle interferometry. ${ }^{25-27}$ Zhang et al. measured in vitro the strain distribution on cartilage and vessel tissues by the digital speckle correlation method (DSCM) and time-sequence speckle pattern interferometry, ${ }^{28}$ and Yao, Wang, and Dai used DSCM to study in vitro damage and fracture progression in cancellous bone. $^{29}$

The complexity of using speckle for dermatological applications stems from the structural complexity and opacity of the skin. Also, in vivo studies involve the nonstationarity of the speckle produced and the need to acquire it in the backscattering configuration. The purpose of this work is to describe significant speckle patterns obtained in vivo from a complex medium such as skin. Therefore, these speckle patterns must be described by extracting quantifiable parameters that discriminate skin lesions, especially radiation burns during the clinically latent period. In this work, a fractal approach is used to describe speckle patterns produced from skin in vivo. Actually, among many methods used to extract information from images of any kind, and in particular medical images, fractal methods are efficient candidates. ${ }^{30-34}$ The flexibility, generality, and robustness of these methods make them adaptable to the treatment of real and complex signals. Of the fractal methods, fractional Brownian motion is commonly used. In a previous study, we showed that speckle patterns from tissue phantoms composed of monodisperse-polystyrene microspheres in solution were well characterized by a fractal approach through the fractional Brownian motion statistic. ${ }^{35}$ Using this approach, three parameters can be extracted from a speckle pattern and characterized on three different scales. These parameters allowed efficient discrimination between different solutions of these tissue phantoms. ${ }^{35}$

In this work, this fractal approach of the speckle was used for the in vivo discrimination of irradiated skin in a porcine model. Speckle patterns from healthy and irradiated porcine skins were generated, and speckle parameters extracted were compared. Series of speckle patterns were recorded several times after irradiation exposure. The efficiency of speckle parameters was tested according to their ability to discriminate irradiated from healthy skin.

\section{Speckle Formation}

Coherent light transmitted by a scattering medium or by a rough surface produces a granular interference pattern, known as a speckle pattern. Statistics of speckle produced from a rough surface have been well known for a long time. ${ }^{36,37}$ In particular, 2-D transverse spatial coherence properties of speckle light are well described in the literature. ${ }^{36-38}$ Typically, the transverse spatial coherence of speckle is described by means of the Van Cittert-Zernike theorem ${ }^{39,40}$ (VCZT), or more exactly by its generalized version, ${ }^{36}$ which accounts for partial coherence of the source. Thus, the transverse correlation function of the intensity distribution at an observation plane is described basically by the Fourier transform of the intensity distribution at the source plane, with a width $d x$ that depends on the distance $L$ to the source plane, on its diameter $d_{e}$, and is on the order of $d x \propto L \lambda / d_{e}$.

However, speckle theory from turbid media is not yet established, and its statistics are notably related to scatterer types in the medium, their distribution, size, and density. In particular, the size of the illuminated area depends on scattering processes in the medium, and can be larger if only its surface is considered. Indeed, it can be considered that photons in a scattering medium, which are characterized by a long path length and so by a large number of scattering events, produce a wide backscattered intensity distribution out of the medium and therefore a large solid angle of illumination resulting in small speckles (VCZT) (see Fig. 1 for illustration). Thus, in the case of tissue phantoms composed of polystyrene-microspheres in solution illuminated in a backscattering configuration and with Monte Carlo simulation, Rajan et al. showed that smaller speckles exhibit a shorter temporal correlation and correspond to photons with longer path length in the medium, compared to larger speckles. ${ }^{41}$ Therefore, we can consider that large speckles mainly correspond to photons that have penetrated slightly in the turbid medium, i.e., near the surface, and can be thus defined by the characteristic size of the illuminating beam. In the same way, small speckles mainly correspond to photons that have undergone significant multiple scattering and can be determined by the whole illuminated area. Hence, the "average width" of a speckle depends on scattering modes present in the medium. The estimation of the speckle dimensions is usually provided by the normalized autocovariance function of the speckle pat- 


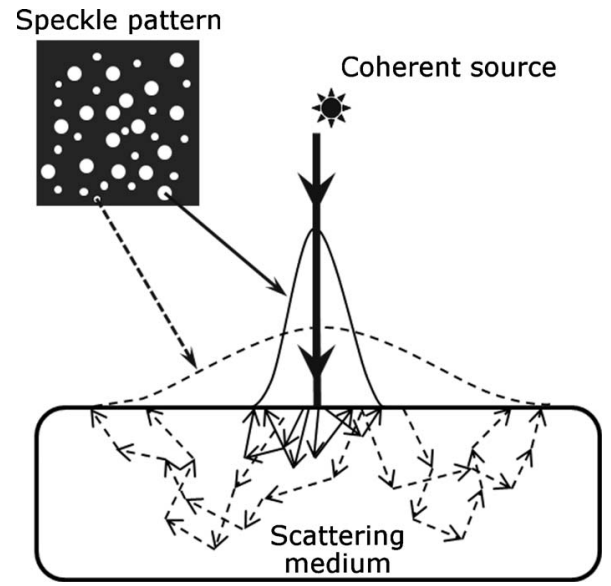

Fig. 1 Short and long path lengths of photons in a scattering medium illuminated in a backscattering configuration provide change in solid angles of illumination, resulting in speckle size variation. ${ }^{41}$

tern intensity received in the observation plane. The width of this function provides a reasonable measurement of "the average width" of a speckle ${ }^{36}$ and is used in many applications. ${ }^{16,42-45}$ Nevertheless, from turbid media illuminated in a backscattering configuration, we can consider that there is a complex spatial interplay of single and multiple scattering that leads to complex spatial fluctuations of intensity of the field formed outside of the medium. This results in a complex spatial distribution of the different coherent areas produced in the observation plane, with a superposition of large and small speckles from single and multiple scattering, respectively. Thus, the multiple-scattering contribution to the intensity cross correlation function can be suppressed by spatially selective detection using the dynamic light scattering technique with a single coherent beam and two closely spaced detectors. Indeed, scattered intensities measured in two points distanced by $\Delta x$ will be correlated only by single scattering, and the multiple-scattering contribution can be neglected if $L \lambda / d_{2} \ll \Delta x \leqslant L \lambda / d_{1}$, where $d_{1}$ and $d_{2}$ are the characteristic size of the single- and multiple-scattering volumes, respectively. A detailed theoretical description of this method was successfully exposed by Lock $^{46}$ for the case of double scattering. An extension of this single-beam cross correlation scheme using a charge-coupled device (CCD) camera as a detector can be also used, as experimentally demonstrated by Zakharov et al. ${ }^{47}$ Therefore, the only measurement of the average width of speckle grains (speckle size) does not seem to be sufficient to take into account this spatial complexity of intensity fluctuations in speckle patterns.

The purpose of this work is not to resolve this problem in a theoretical way. Our aim is to describe efficiently speckle patterns obtained experimentally from a complex medium such as skin to discriminate skin lesions in the case of radiation burns, especially during the clinically latent period. For this purpose, we chose a fractal approach through fractional Brownian motion to characterize speckle patterns.

\section{Speckle Pattern Processing}

Fractal methods are efficient candidates for extracting information from complex images such as biomedical images, for example, to analyze osteoporosis or cerebral pathologies. ${ }^{30-33}$ Speckle pattern can be considered a complex image due to the difficulty in describing it mathematically. ${ }^{48}$ Thus, fractal approaches can be appropriate in the study of the behavior of a chaotic system such as speckle pattern. The flexibility, generality, and robustness of these approaches could make them adaptable to the treatment of such complex signals. Nevertheless, fractal properties in speckle pattern are usually observed from fractal objects ${ }^{49-52}$ and such speckles are called "fractal speckles."

However, in the case of a scattering surface, several authors found fractal properties in speckle patterns from nonfractal objects. Hence, theses speckles cannot be called fractal speckles. For example, fractal properties in speckle patterns were found in the case of a diffuser illuminated with a ringslit aperture, ${ }^{49,51}$ or in the case of several illuminated materials and bones studied by speckle interferometry in backscattering configuration. $^{48,53}$ Fractal properties were also found in speckle patterns from metallic standard-rough surfaces, and the fractal dimension of speckle patterns according to surface roughness and styles was calculated. ${ }^{54}$

In addition, in the case of scattering volume media, fractal properties were found in speckle patterns from nonfractal media. For example, fractal properties were found in the region of small time scales in the case of dynamic biospeckles, and the 1-D fractional Brownian process was used for the description of the dynamic speckle-intensity fluctuations. ${ }^{55}$ Although their study concerns dynamic speckle, in their point of view the spatial distributions of backscattered fields formed by turbid media can be interpreted as random 2-D structures that demonstrate fractal properties in the region of small spatial scales. ${ }^{55}$ Likewise, from tissue phantoms composed of polystyrene microspheres in solution illuminated in a backscattering configuration, fractal properties in speckle patterns were found in high-frequency ranges by a decrease in $1 / f$ of their power spectral density (PSD), ${ }^{35,36}$ which is characteristic of a self-similar process. ${ }^{57}$ In particular, in our previous work, optical properties of skin and its complex composition were represented by such tissue phantoms, notably from several mixtures of different sized microspheres in different proportions. $^{35}$

Thus, according to all these references, and in particular according to the previous work, ${ }^{35}$ we expected to find fractal properties in the speckle field from biological tissues such as skin, and we considered that we can apply a fractal approach to analyze the speckle patterns observed.

Consider a speckle pattern where $I(x, y)$ is the intensity in gray-level value of the pixel located at coordinates $(x, y)$. For a given horizontal direction $y$ of the speckle pattern, and assuming second-order stationarity, the second-order structure function of the intensity $D_{2}(\Delta x)$ can be expressed as:

$$
\begin{aligned}
D_{2}(\Delta x) & =\left\langle[I(x+\Delta x, y)-I(x, y)]^{2}\right\rangle \\
& =2\left\{\left\langle I(x, y)^{2}\right\rangle-\langle I(x+\Delta x, y) I(x, y)\rangle\right\},
\end{aligned}
$$

where $\Delta x$ is the distance between two pixels and \langle\rangle is the spatial average. Then: 


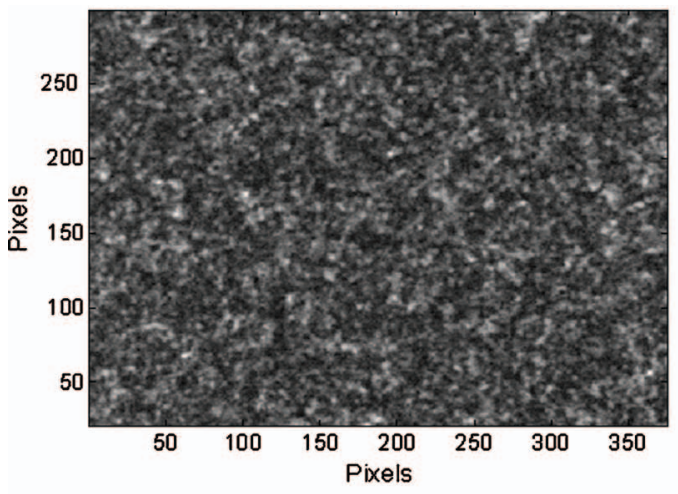

(a)

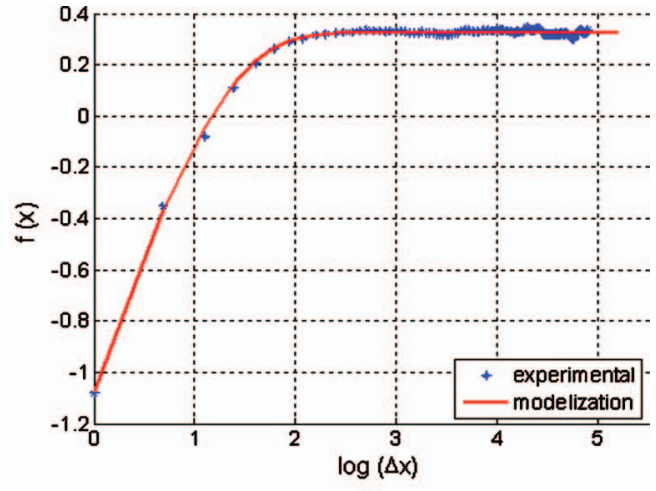

(b)

Fig. 2 Speckle pattern obtained from healthy porcine skin for observation angle (a) $\psi=20$ deg and (b) corresponding diffusion curve.

$$
D_{2}(\Delta x)=2\left\{\left\langle I(x, y)^{2}\right\rangle-C_{f f}(\Delta x)\right\}
$$

where $C_{f f}(\Delta x)=\langle I(x+\Delta x, y) I(x, y)\rangle$ is the autocorrelation function of the speckle pattern intensity $I$, and $\left\langle I(x, y)^{2}\right\rangle=\sigma_{I}^{2}$ $+\langle I(x, y)\rangle^{2}$.

Previous studies have shown that experimental speckle patterns present fractal properties by their self-similar behavior in the high-frequencies domain. ${ }^{35,54,56}$ From fractal theory, ${ }^{57}$ a process $X$ that presents such behavior according to the variable $t$ is described for its autocorrelation function by:

$$
C_{f f}(\Delta t)=\langle X(t) X(t+\Delta t)\rangle=\sigma^{2} \exp \left(-\lambda|\Delta t|^{2 H}\right),
$$

where $H \in[0,1]$ is the Hurst coefficient that reflects the Hölderian regularity in each point of the process. ${ }^{58}$

Hence, from Eqs. (2) and (3) and after centering the process, the second-order structure function in speckle pattern, for every horizontal dimension $y$, can be expressed as:

$$
D_{2}(\Delta x)=2 \sigma_{I}^{2}\left[1-\exp \left(-\lambda|\Delta x|^{2 H}\right)\right] .
$$

Then:

$$
\log \left[D_{2}(\Delta x)\right]=\log \left(2 \sigma_{I}^{2}\right)+\log \left[1-\exp \left(-\lambda|\Delta x|^{2 H}\right)\right] .
$$

Three parameters can be extracted from this equation:

- $H$, the Hurst coefficient, is related to the image on a local scale. Indeed, it can be easily shown that if $\Delta x \ll \lambda$, i.e., on a local scale in the speckle pattern, and for $H>0.5$, Eq. (5) can be written as:

$$
\log \left[D_{2}(\Delta x)\right] \propto 2 H \log (\Delta x) .
$$

The increment process described in Eq. (6) is analogous to fractional Brownian motion one. ${ }^{58}$ Equation (6) is known as the diffusion function of the intensity in the direction $x$.

- $S$, the self-similar element, given by ${ }^{59} \pi / \lambda$, allows for the quantization of the dimension in the image that separates the classic from the self-similarity properties of the speckle. In this dimension, the process is scale invariant.

- $G$, the saturation of the variance equal to $2 \sigma_{I}^{2}$, characterizes the image on a global scale.
For a speckle pattern and for every horizontal dimension $y$, the logarithm of the second-order structure function $f(x)$ $=\log \left[D_{2}(\Delta x)\right]$ is calculated and approximated according to Eq. (5) by:

$$
\left\|f(x)-\left\{a+\log \left[1-\exp \left(-b|\Delta x|^{c}\right)\right]\right\}\right\|_{2}^{2} \leqslant \varepsilon,
$$

where $\varepsilon=10^{-5}$ is a mean square error. We can then identify the three coefficients $a, b, c$ according to Eq. (5):

$$
\left\{\begin{array}{l}
a=\log \left(2 \sigma_{I}^{2}\right) \\
b=\lambda \\
c=2 H
\end{array} .\right.
$$

One speckle pattern is treated row by row or column by column. The average value of the intensity was subtracted from all intensity values for image normalization before processing. After using the previous processing procedure and for each dimension in the image, the mean of a given parameter is calculated for all rows or columns and is used to characterize the speckle pattern for further comparisons.

Figure 2 illustrates an example of a speckle pattern obtained from healthy porcine skin for the observation angle $\psi=20 \mathrm{deg}$ [Fig. 2(a)], and the graphical result of the processing described before for the horizontal direction [Fig. 2(b)]. The corresponding PSD is presented in Fig. 3. Thus in the PSD of the speckle pattern, self-similar behavior can be observed in high-frequency range by a decrease in $1 / f$, as observed in the previous work. ${ }^{35}$ This behavior can also be observed in the second-order structure function of the speckle pattern intensity in small spatial scales, in the same range of 1 to 2 decades [Fig. 2(b)]. Moreover, according to Fig. 2(b), Eq. (7) allows a good fitting of the second-order structure function.

Due to the presence of multiscale behavior observed in the PSD of speckle patterns, the structure function of the intensity $D_{2}(\Delta x)$ is appropriate for analysis of the speckle pattern intensity because it displays prominently the intensity fluctuations on several spatial scales. Thus, we can see that the variation of spatial intensity fluctuations is not the same on a global scale (far neighborhood or large spatial scales) and on a local scale (near spatial neighborhood or small spatial 


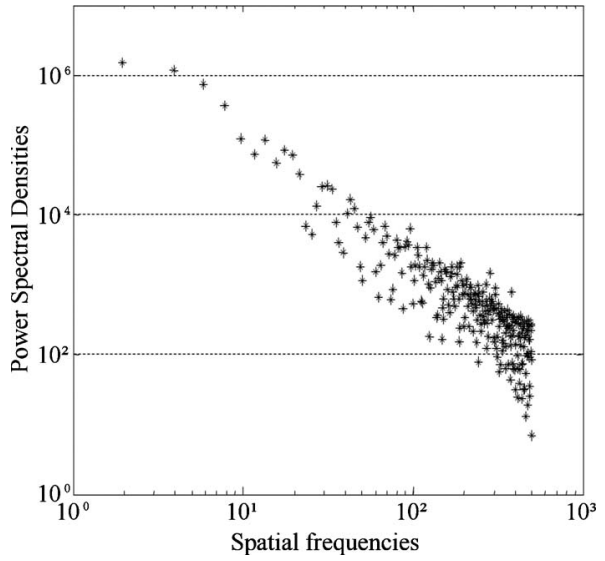

Fig. 3 Power spectral density of the speckle pattern presented in Fig. 2(a).

scales) where self-similarity is observed [Fig 2(b)]. Thus, the second-order structure function takes into account the multiscale aspect of the speckle.

From the second-order structure function, two major parts can be observed: the part at small spatial scales where selfsimilar behavior is observed, and the part at the far neighborhood, where the intensity fluctuations become approximately constant. The last part can be characterized by the saturation of the variance $G$ [Eq. (5)]. The first part can also be divided in two parts: a very local scale, in the vicinity of zero (when $\Delta x \rightarrow 0$ ), characterized by the Hurst coefficient $H$ [Eq. (6)]; and an intermediate scale, which separates the two behaviors (self-similar and classical behaviors). The self-similar element $S$ characterizes the dimension in speckle pattern that separates the classic from the self-similar behaviors. Because $G$ characterizes the speckle pattern statistic at a global spatial scale, where no self-similar behavior is observed, it cannot be considered a fractal parameter. Hence, another advantage of Eq. (7) is that three parameters can be extracted corresponding to three different scales in the speckle pattern, and can thus correspond to different properties in skin. Also, according to Eqs. (3) and (4), the parameters used constitute a mathematical parameterization of the autocorrelation function of the speckle that allows extraction of more parameters than the speckle size. $^{35}$

A few studies have shown fractal properties in cell structures and tissues. ${ }^{60-62}$ Although there is no proof that the volume of the skin crossed by the coherent light has fractal properties (so we cannot call the speckle observed "fractal speckle"), fractal properties were found in high-frequency range from speckle patterns produced. From our point of view, in turbid media illuminated in a backscattering configuration, the spatial structure of the field formed outside of the medium is complicated by the presence of several scattering modes in the medium. Hence, the interplay of single and multiple scattering (corresponding essentially to surface and volume scattering, respectively) could lead to complex spatial intensity fluctuations, resulting in speckles with fractal properties in small spatial scales. Thus, for Zimnyakov et al., a parameter that describes scaling properties of the intensity fluctuations (Hurst coefficient or fractal dimension) is very sensitive to changes in the optical properties of turbid media.
The Hurst coefficient depends notably on the scattering geometry and scattering modes present in the medium, and can be used for the identification of various scattering modes and processes. ${ }^{55}$ Even though their study concerns dynamic fluctuations of speckle intensity, they consider (in an analog way) that the complex spatial distributions of backscattered fields formed by scattering processes show fractal properties in the region of small spatial scales. ${ }^{55}$ Therefore, in that case, the Hurst coefficient $H$ extracted by Eq. (8) could depend on scattering processes present in the medium and thus on the density of scatterers or on their distribution in size.

\section{Experimental Procedure}

\subsection{Irradiation Protocol}

Porcine skin is the reference biological model of human skin because of its significant anatomical and physiological similarities. ${ }^{11}$ Two female minipigs of the Pitman-Moore strain of about $20 \mathrm{~kg}$ and 6 months of age were used. According to the age of animals throughout the experiments, structure and function of minipig skin can be considered as invariant with time under normal conditions, i.e., without applying any stress. ${ }^{11}$ Pigs were locally irradiated with $40 \mathrm{~Gy}$ of gamma radiation [60Co, IRDI 4000, Centre de Recherche du Service de Santé des Armées (CRSSA), Grenoble, France] with a rate of $1 \mathrm{~Gy} / \mathrm{min}$, on an area of $5 \times 10 \mathrm{~cm}^{2}$ in the right-side dorsal zone. The position of the animals allowed an irradiation perpendicular to the animal's spinal axis. To avoid irradiating the spinal column and to limit intestine exposure, the irradiated area was located a few centimeters below the spine. A 1-cm-thick wax block was positioned on the irradiated skin to achieve electronic balance conditions in the skin and thus obtain homogeneity of the dose at depth.

To control the doses delivered to the skin, four thermoluminescent dosimeters of alumina powder $\left(\mathrm{Al}_{2} \mathrm{O}_{3}\right)$ were incorporated into the wax used, three at the surface directly exposed and one on the surface opposed.

After irradiation, pigs were tattooed on two areas for measurements, in the irradiated zone and in the healthy zone. Each animal was taken as its own control. Because skin structure varies according to the location on the body, ${ }^{11}$ the control cutaneous zone chosen must be similar to the irradiated zone for comparison. Hence, the irradiated and healthy skin areas chosen are both located in the right-side dorsal zone. The distance between the two cutaneous areas was about $30 \mathrm{~cm}$. Experiments were conducted in compliance with French regulations for animal experimentation (Ministry of Agriculture, Act 87-848, 19 October 1987), and approved by the Institut de Radioprotection et de Sûreté Nucléaire (IRSN) ethics committee.

\subsection{Speckle Measurement}

The experimental setup to observe backscattering speckle produced by porcine skin consisted of a nonpolarized HeNe laser (Melles Griot, Albuquerque, New Mexico, 05-LHR-171), a CCD camera (Kappa, Los Angeles, CF 8/1 DX), and a mechanical guide and processing software for the image processing (MatLab, Mathworks, Natick, Massachusetts). The laser illuminates the skin at $632.8 \mathrm{~nm}$ with an intensity power of $15 \mathrm{~mW}$, and the CCD camera contains $384 \times 288$ pixels of 


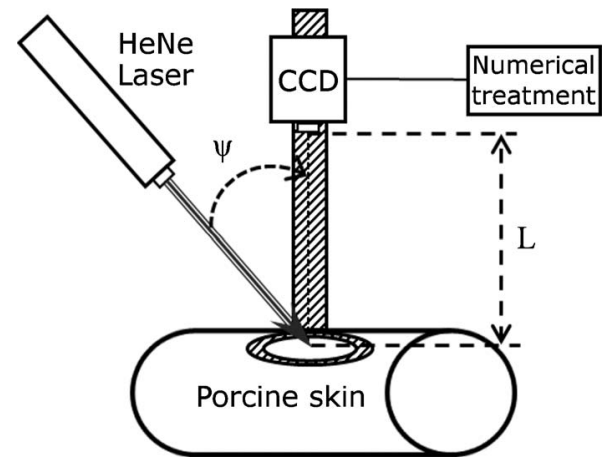

Fig. 4 Experimental setup for in vivo acquisition of speckle patterns from skin.

size $8 \times 8 \mu \mathrm{m}$. The mechanical guide contains an open torus that is in contact with skin. The torus aperture is about $40 \mathrm{~mm}$ in diameter, which allows skin illumination by laser beam without parasite reflections. The mechanical guide keeps the CCD-skin distance constant between acquisitions and softens movements of the animal due to respiration or other factors caused by anesthesia. The CCD camera, mechanical guide, and laser were placed in the vertical plane; the surface of the skin was placed in the horizontal plane (Fig. 4). The CCD camera and the guide were placed in the normal direction at the skin surface. Given the measurement geometry used in this work, we would expect to observe deformed speckles, i.e., nonuniform speckles along the two dimensions in the speckle pattern. Indeed, illumination angles lead to a form of the illuminated area more elliptical than circular (VCZT). However, the speckle pattern seems quite uniform in Fig. 2(a). This can be explained by the small illumination angle used $(\psi=20 \mathrm{deg})$.

Speckle size increases with distance $L$ between the surface of the medium and the CCD camera. ${ }^{63}$ To have a good statistical evaluation of the speckle pattern, the image must have many speckle grains. Furthermore, speckle size must be larger than the pixel size of the CCD array. ${ }^{64}$ To respect both conditions, we set the CCD camera at a distance $L=20 \mathrm{~cm}$ from the skin surface.

The HeNe laser was positioned at an angle $\psi>10 \mathrm{deg}$ from the CCD camera to avoid recording specular reflections from the skin surface. All experiments were carried out at three observation angles $\psi: 20,40$, and $60 \mathrm{deg}$. The more the speckle pattern acquisition is carried out outside the specular reflection, the more information located deep in the skin is acquired.

Since speckle acquisition is done in vivo, physiological factors such as heart rate, blood and lymph flows, microcirculation, or breathing involve mobility in the medium. Therefore, random agitation of the speckle intensity is observed, which is called "boiling speckle" and corresponds to the speckle intensity fluctuations resulting in a loss of correlation in speckle pattern. To avoid recording this blurred speckle, the exposure time of the camera must be shorter than the time scale of these fluctuations. ${ }^{24}$ We chose the shortest image acquisition time provided by our camera of $1 \times 10^{-4} \mathrm{~s}$. To take into account spatial physiological heterogeneities, eight tattooed points were measured in the irradiated and nonirradi- ated cutaneous zones in each experiment. For each day of experiments and for each point measured in one cutaneous zone, 200 speckle patterns were recorded by the CCD camera with $25-\mathrm{Hz}$ frequency, and were digitized with 8-bit precision by the analog-to-digital converter. About 9600 speckle patterns were then recorded in one day of experiments.

Since we did not know the duration of the clinically latent period, experiments were carried out once a week after irradiation and until serious clinical signs appeared.

\subsection{Data Analysis}

The large data sample $(n=200)$ from each point measured in skin allowed us to carry out the ANOVA test ${ }^{65}$ to emphasize discrimination between cutaneous zones (groups). To compare variability between the eight points measured for the same zone and the variability between zones, we applied the twofactor ANOVA test. We defined the parameter $p_{A}$, the $p$-value for the null hypothesis $H_{0 A}$, corresponding to factor $A$ (variability interzone), and parameter $p_{B}$, the $p$-value for the null hypothesis $H_{0 B}$, corresponding to factor $B$ (variability intrazone). For all data, discrimination was established significantly if the $p$-value was less than 0.01 .

Principal component analysis ${ }^{66}$ was carried out to give an overview of all results, taking into account their dispersion. This analysis allowed us to assess the homogeneity and coherence of data and the correlation between variables. Each line of the input data matrix corresponded to the values of $H$, $G$, and $S$ of each speckle pattern acquired, for all observation angles. The input data matrix was thus comprised of nine variables ( $H, S$, and $G$ for the three angles) and of 3200 lines (number of observations for all cutaneous zones).

\section{Results}

The model parameters were calculated for each speckle pattern recorded, according to the procedure described in Sec. 2. The horizontal direction of the speckle pattern, which contains the highest number of pixels, was chosen for treatment.

Tables 1 and 2 present, for all experiments, the stochastic parameters that discriminate significantly irradiated versus healthy zones for pigs named $\mathrm{P} 1$ and $\mathrm{P} 2$, respectively $\left(p_{A}\right.$ $<0.01)$. For each discriminant result, the two-factor ANOVA test showed $p$-values such as $p_{A} \ll p_{B}$, which means that the greatest dispersion of measurements is statistically caused by the difference between the healthy and irradiated zones (factor $A)$. Clinical signs are also given in these tables. Time is coded so that $D$ gives days after ionizing radiation exposure, and $d$ gives days where clinical signs appeared. According to the clinical course in humans, ${ }^{11}$ a clinically latent postirradiation period was observed in the two pigs. The first clinical sign observed was cutaneous sensitivity at D93 for pig P1 and edema at D78 for pig P2. Some differences between the pigs were noted in the kinetic of lesion emergence and lesion severity. As in humans, differences in clinical responses between the pigs could be explained by differences in individual radiosensitivity. Pig P1 was euthanized at D112 to end unrelievable suffering. Lesion chronology observed for pig P2 is typical of the known chronology of radiation-induced damage in humans. ${ }^{11}$ Once skin lesions emerged in pig P2, measurements were devoted to undamaged skin areas, as the aim was not to make measurements directly on an apparent lesion, 
Table 1 Speckle parameters that discriminate between irradiated and healthy cutaneous zones ( $p$ $<0.01)$ for pig P1 according to time and clinical injury observed.

\begin{tabular}{|c|c|c|c|c|c|}
\hline $\begin{array}{l}\text { P1 } \\
\text { Days after } \\
\text { radiation } \\
\text { exposure }\end{array}$ & $\psi=20 \mathrm{deg}$ & $\psi=40 \mathrm{deg}$ & $\psi=60 \mathrm{deg}$ & Clinical injury & $\begin{array}{c}\text { Days before } \\
\text { injury emergence }\end{array}$ \\
\hline D15 to D55 & - & - & - & - & $d-78$ to $d-38$ \\
\hline D64 & $G, H$ & $G, H$ & $G, H$ & - & $d-29$ \\
\hline D75 & $G, H$ & $G, H$ & $G, H$ & - & $d-18$ \\
\hline D84 & $H$ & $G$ & $H$ & - & $d-9$ \\
\hline D93 & $H$ & $H$ & - & Skin sensitivity & $d$ \\
\hline D104 & $H$ & - & $H$ & - & $d+11$ \\
\hline D1 12 & $H$ & - & $H$ & Suffering & $d+19$ \\
\hline
\end{tabular}

which necessarily alters the speckle statistics and for which discrimination can be done visually. Extension of the damaged area in pig P2 (Fig. 5) led to interruption of experiments on D106.

Discrimination between healthy and irradiated skin was effective for 29 days and 20 days before emergence of clinical signs for pigs P1 and P2, respectively. During this latent period characterized by the absence of clinical signs, two parameters allowed discrimination for both pigs: the Hurst coefficient $H$ and the saturation of the variance $G$. The selfsimilar element discriminates only when serious lesions have appeared (Tables 1 and 2). Hence, this parameter does not seem to be relevant for early discrimination between irradi- ated and healthy skin. Table 3 presents the score of discriminant parameters calculated for all times and for each observation angle $\psi$. For each pig, the Hurst coefficient $(H)$ seems to be the most effective parameter for discrimination between irradiated and healthy skin for the angles $\psi=20 \mathrm{deg}$ and $\psi$ $=60 \mathrm{deg}$ (score of 10 and 12, respectively). Moreover, the angle $\psi=40$ deg seems to be the least relevant for discrimination for all parameters.

For each parameter and each experimental configuration, mean and standard deviation values of 1600 speckle patterns recorded in a cutaneous zone were deduced. Figure 6 illustrates the variation with time of the Hurst coefficient $(H)$ for

Table 2 Speckle parameters that discriminate between irradiated and healthy cutaneous zones ( $p$ $<0.01)$ for pig P2 according to time and clinical injury observed.

\begin{tabular}{|c|c|c|c|c|c|}
\hline $\begin{array}{l}\text { P2 } \\
\text { Days after } \\
\text { radiation } \\
\text { exposure }\end{array}$ & $\psi=20 \mathrm{deg}$ & $\psi=40$ deg & $\psi=60 \mathrm{deg}$ & Clinical injury & $\begin{array}{l}\text { Days before } \\
\text { injury emergence }\end{array}$ \\
\hline D8 to D52 & - & - & - & - & $d-70$ to $d-26$ \\
\hline D58 & - & - & $G, H$ & - & $d-20$ \\
\hline D69 & $G$ & $G$ & $G, H$ & - & $d-9$ \\
\hline D78 & $H$ & $H$ & $H$ & Edema & $d$ \\
\hline D84 & $H$ & - & $H$ & - & $d+6$ \\
\hline D92 & - & - & $H$ & $\begin{array}{l}\text { Hyperkeratosis, } \\
\text { beginning of dry } \\
\text { desquamation }\end{array}$ & $d+14$ \\
\hline D99 & $H, S$ & - & $H, S$ & $\begin{array}{c}\text { Moist } \\
\text { squamation }\end{array}$ & $d+21$ \\
\hline D106 & $H, S$ & $G, H, S$ & $G, H, S$ & $\begin{array}{l}\text { Beginning of } \\
\text { necrosis }\end{array}$ & $d+28$ \\
\hline
\end{tabular}




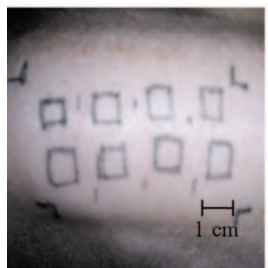

(a)

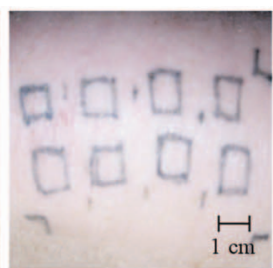

(b)

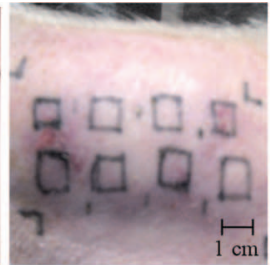

(c)

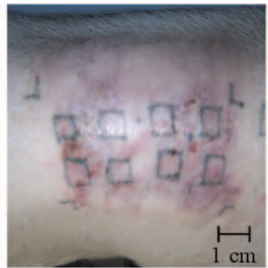

(d)

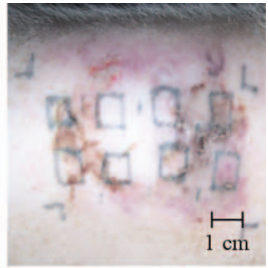

(e)

Fig. 5 Clinical course of the irradiated skin area for pig P2 at (a) D69, (b) D84, (c) D92, (d) D99, and (e) D106.

irradiated (continuous red lines) and healthy zones (dashed blue lines) for $\psi=20$ and $60 \mathrm{deg}$ for pigs P1 and P2, respectively. In these two cases, a decrease in $H$ was observed with time for the irradiated skin. The ANOVA test followed by a multicomparison test was used to check if the healthy zone changed statistically with time (with an error of less than $0.1 \%$ ). For pig P2, the multicomparison test showed that the healthy zone was statistically similar for all times, and for all times except for D55 $(p=0.0005)$ for pig P1. This time point is the only atypical point observed. The significant difference of the healthy zone at this time compared with other times may have led to nondiscrimination between irradiated and healthy zones. Thus, we can consider that healthy skin did not lead to significant variations in the speckle produced with time.

Figure 7 illustrates the results of the principal component analysis (PCA) for several times for the pig P2 example. Variables and observations are presented by vectors and point clouds, respectively, on the same graph in the principal plane; red and blue clouds correspond to observations in irradiated and healthy zones, respectively. According to the PCA, the two point clouds corresponding to the healthy and irradiated zones were separating gradually (at D22 to D106), with a greater dispersion for the irradiated zone. Similar results were obtained for pig P1.

\section{Discussion}

For the first time, in vivo discrimination of radiation burns during the clinically latent period, when no sign is yet visible, is possible using a noninvasive method. Therefore, the use of the speckle phenomenon is promising to the diagnosis and prognosis of radiation skin injuries. This technique could contribute to real progress in the medical care of patients locally overexposed to ionizing radiation, since earlier diagnosis will improve treatment effectiveness.

\subsection{Dose Selection}

All experiments were carried out on live pigs. Housing and care of pigs in an animal facility is demanding logistically and time-wise, and euthanasia is ethically required if suffering cannot be stopped, even if the experiments are not complete. For these reasons, only one radiation dose was used in our experiments.

To provide lesion prognosis after radiation exposure, it was necessary to wait for clinical signs to appear. The dose threshold for appearance of clinical signs in humans is 10 to $15 \mathrm{~Gy}$ for dry desquamation and 16 to $20 \mathrm{~Gy}$ for moist desquamation. ${ }^{11}$ Skin necrosis appears in humans from a dose threshold of 20 to $25 \mathrm{~Gy}$. ${ }^{11}$ The appearance of necrosis is crucial for clinicians, as it indicates the need to excise tissues to prevent its spread. It is therefore important to prevent skin necrosis emergence. Several radiation doses were previously administered to several pigs to reproduce such a skin lesion. A dose of $40 \mathrm{~Gy}$ was required in our experimental conditions to obtain skin necrosis, a dose twice that required to generate this lesion in humans. The difference observed in clinical sensitivity between pigs and humans may relate to the location of the irradiated zone selected in our experiments. Indeed, the

Table 3 Score of discriminant parameters, calculated for all times, for each observation angle, as well as for each pig and for both pigs.

\begin{tabular}{|c|c|c|c|c|c|c|c|c|c|}
\hline \multirow[b]{2}{*}{ Pig } & \multicolumn{3}{|c|}{$\Psi=20 \mathrm{deg}$} & \multicolumn{3}{|c|}{$\Psi=40 \mathrm{deg}$} & \multicolumn{3}{|c|}{$\Psi=60 \mathrm{deg}$} \\
\hline & $G$ & $H$ & $S$ & $G$ & $H$ & $S$ & $G$ & $H$ & $S$ \\
\hline \multirow[t]{2}{*}{ P1 } & 2 & 6 & 0 & 3 & 3 & 0 & 2 & 5 & 0 \\
\hline & \multicolumn{3}{|c|}{ Total $=8$} & \multicolumn{3}{|c|}{ Total $=6$} & \multicolumn{3}{|c|}{ Total $=7$} \\
\hline \multirow[t]{2}{*}{ P2 } & 1 & 4 & 2 & 2 & 2 & 1 & 3 & 7 & 2 \\
\hline & \multicolumn{3}{|c|}{ Total $=7$} & \multicolumn{3}{|c|}{ Total $=5$} & \multicolumn{3}{|c|}{ Total $=12$} \\
\hline \multirow{2}{*}{$\begin{array}{l}\text { Total for both } \\
\text { pigs }\end{array}$} & 3 & 10 & 2 & 5 & 5 & 1 & 5 & 12 & 2 \\
\hline & \multicolumn{3}{|c|}{ Total $=15$} & \multicolumn{3}{|c|}{ Total $=11$} & \multicolumn{3}{|c|}{ Total $=19$} \\
\hline
\end{tabular}




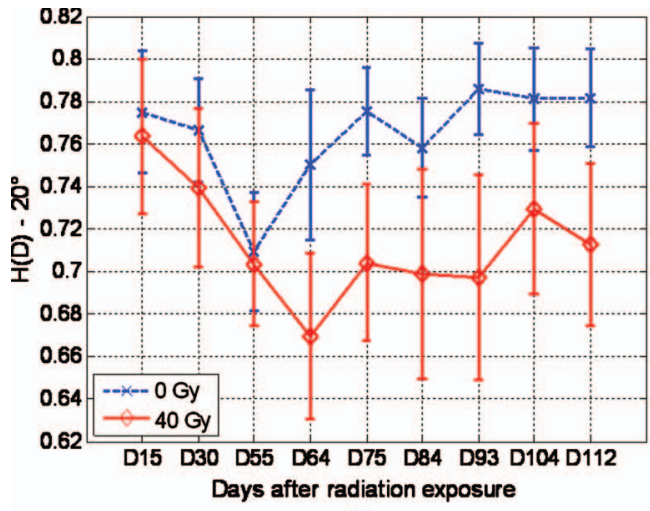

(a)

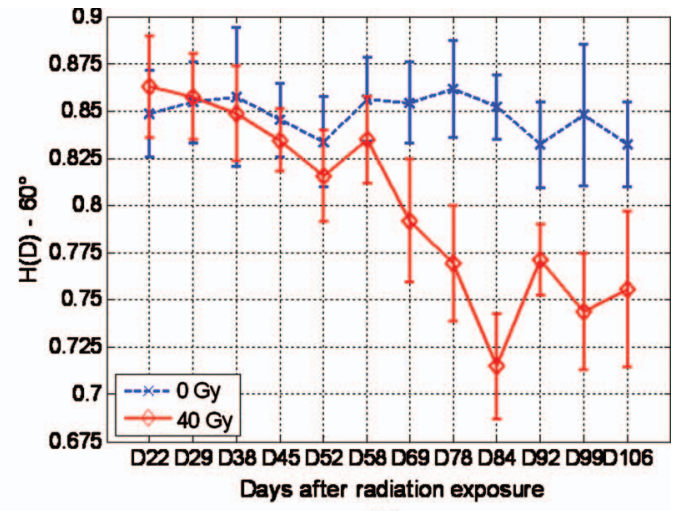

(b)

Fig. 6 Hurst coefficient as a function of time after irradiation, for irradiated (continuous red line) and healthy (dashed blue line) cutaneous zones for (a) pig P1 and $\Psi=20$ deg and (b) pig P1 and $\Psi=60$ deg.

pig skin area most similar to human skin is the inner thigh, but it was not possible to irradiate this area because of friction between the thighs generated during the animal's daily movements, as this could weaken the irradiated cutaneous zone. Porcine skin in the dorsal zone is thicker, and that is probably why clinical signs only appeared at $40 \mathrm{~Gy}$. Other experiments are needed to confirm these observations with $40 \mathrm{~Gy}$.

\subsection{Discrimination}

For the porcine models under study, and notably during the clinically latent postirradiation period, the Hurst coefficient $H$ and the saturation of the variance $G$ seem to be the most effective parameters for discrimination between irradiated and healthy skin (Tables 1 and 2). Early discrimination of speckle patterns on a global scale by the saturation of the variance and on a local scale by the Hurst coefficient shows that discrimination occurs on two scales of speckle patterns. The selfsimilar element $S$ allows discrimination only when serious clinical lesions emerge in pig P2 (Table 2). This would mean that $S$ is not connected to the same properties in skin than $H$ or $G$, and so it would bring different information about skin changes. Thus, when integrating the multiscale aspect of the speckle pattern, the fractal approach used would provide complementary information about speckle pattern, and thus about skin changes.

For both animals, the parameter $H$ seems to be the most efficient because of its relevance in discrimination for $\psi=20$ and $60 \mathrm{deg}$ (Table 3). The Hurst coefficient tended to decrease with time after irradiation for both pigs (Fig. 6). For pig P2, this decrease seemed to be intensified until D84, just before more serious lesions appeared. After this time, $H$ values increased, so there was less difference between the two cutaneous zones. We can suppose that this variation is related to the animal's reaction to radiation stress, which can be characterized by dominance of the healing process in the skin. The efficiency of the Hurst coefficient for discrimination shows that it is very sensitive to changes in optical properties in a turbid media such as skin. A high sensitivity of this parameter in turbid media was also observed in the case of dynamic biospeckles. $^{55}$

Data homogeneity and coherence were observed in the PCA representation when discrimination was available (Fig.
7). Hence, discrimination between irradiated and healthy zones is not caused by heterogeneity between points of measurements. The two measured cutaneous zones are located in the same region of the pig. Moreover, speckle from the healthy zone remains relatively constant with time according to the multicomparison test. Hence, the separation between the two point clouds from D22 to D106 reflects that the skin property changes are in the irradiated zone. Therefore, the dose is the factor most likely to explain the discrimination observed between healthy and irradiated zones. More data from animal experiments could be helpful in reinforcing these first and promising results.

Discrimination by four variables for D69 and D99 (Table 2) can explain the distinction between the two point clouds in the PCA representation [Figs. 7(c) and 7(e)]. A distinction between the two clouds was also observed at D92, although only $H$ discriminates for $\psi=60 \mathrm{deg}$ with a risk of error of $1 \%$ according to the previous ANOVA test (Table 2). The distinction observed at this time can be explained by the strong contribution to the discrimination of three other variables $G$, $S$, and $H$ for $\psi=40 \mathrm{deg}$ in this representation [Fig. 7(d)]. Their very low $p_{A}$-values justify their contribution $\left(p_{A}\right.$ $=0.014 ; p_{B}=0.86, p_{A}=0.013 ; p_{B}=0.39$ and $p_{A}=0.012 ; p_{B}$ $=0.70$ for $H, G$, and $S$, respectively). Likewise, four variables can be considered as discriminating at D78, which can explain the distinction between the two point clouds at this time (data not shown). Hence, we can consider that at D92 and D78, like at D69 or D99, at least four variables seem to be required to distinguish irradiated from healthy zones in this PCA representation. Discrimination by the three angles for several parameters at D106 (Table 2) explains the total separation between the clouds representing the two zones [Fig. 7(f)]. Moreover, the PCA representation shows an anticorrelation between the Hurst coefficient on the one hand and $S$ and $G$ on the other hand: $H$ decreased with radiation, whereas $G$ and $S$ increased. Consequently, it would be possible to define a parameter from a combination of all the variables used, which could allow more effective (that is, earlier) discrimination.

Discrimination during the clinically latent postirradiation period shows the potential of the technique presented in this work in terms of early diagnosis and prognosis. Nevertheless, 


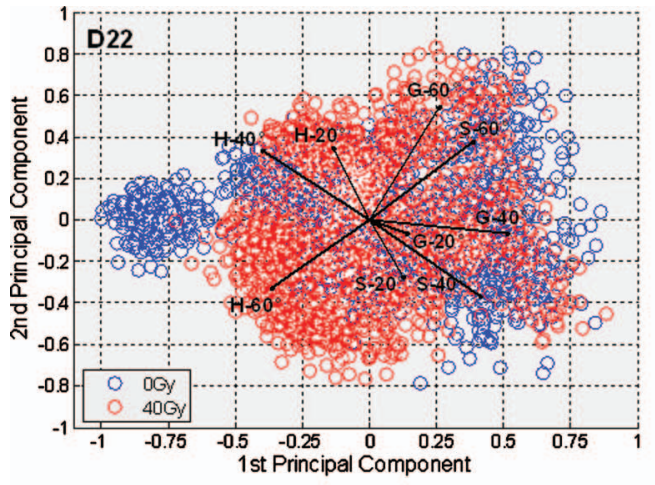

(a)

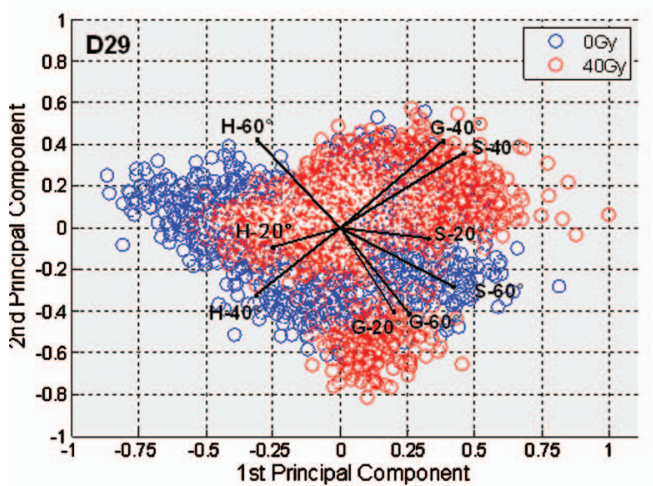

(b)

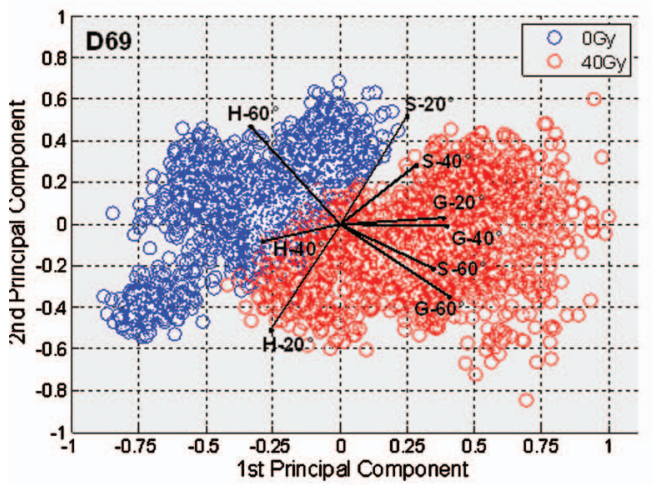

(c)

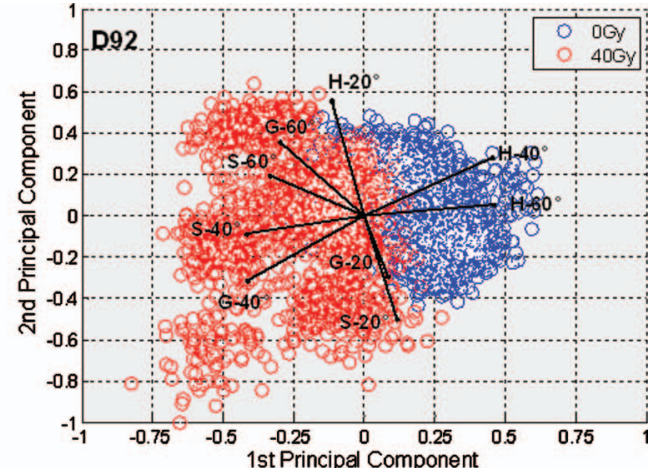

(d)

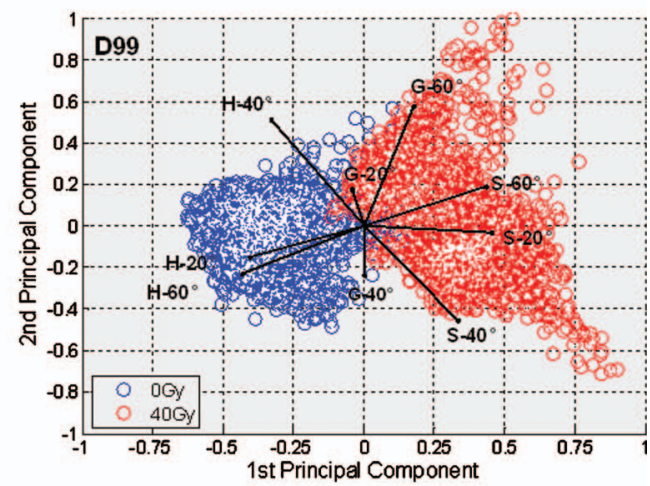

(e)

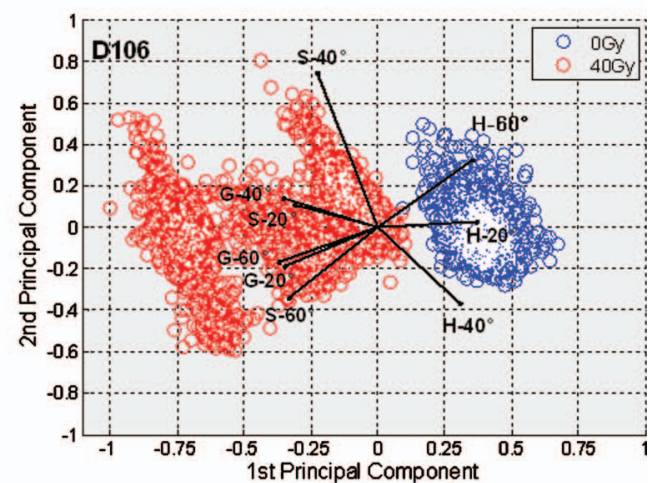

(f)

Fig. 7 Principal component analysis (PCA) representation for pig P2 several times after irradiation. Variables are represented by black vectors (nine variables: $G, H, S$ for three observation angles) in the same graph of observations (red and blue point clouds corresponding to irradiated and healthy zones, respectively).

discrimination only 29 and 20 days before emergence of the first clinical sign for pigs $\mathrm{P} 1$ and $\mathrm{P} 2$, respectively, can be limiting. Indeed, if the method sensitivity is lower in humans than in pigs, it will be important to discriminate irradiated porcine skin at the earliest after radiation exposure. The low performance of the CCD camera used in this work probably affected the sensitivity of parameters for discrimination. This could explain why the discrimination was only significant from D64 and D58 after radiation exposure for pigs P1 and $\mathrm{P} 2$, respectively. To achieve earlier discrimination with speckle data, a shorter exposure time may be required to minimize the loss of correlation induced by intensity fluctuations. In addition, a CCD camera with a significantly higher image resolution could be used to provide higher spatial resolution in speckle patterns, which may allow improved measurements of intensity increments on the local scale. A higher dynamic range of the camera could be used to give a higher gray-level resolution that could also improve the measurement of the second-order structure function. For the overall parameters and pigs, the poor relevance in discrimination of the observation angle $\psi=40 \mathrm{deg}$ could indicate that the skin changes detected are mainly located in surface layers and deep layers rather than in middle layers. To complete the skin exploration at different depths, polarizers could be used to observe speckles at different states of polarization in different illumination 
configurations: perpendicular or parallel polarization states in linear or circular incident illumination. ${ }^{67}$

\subsection{Interpretation of Parameter Variations_-First Hypotheses}

The classical frequential approach, based on calculation of the normalized autocovariance function, characterizes the speckle pattern spatially by providing a reasonable measurement of the "average width" of speckle grains, ${ }^{36}$ usually called speckle size. The self-similar element $S$ gives the dimension in the image of the self-similarity behavior, which is in the grain neighborhood. Hence, speckle size and the self-similar element are both statistically related to the mean dimension of the speckle grain. ${ }^{35}$ Similar variations between these parameters were thus observed with scattering size change, ${ }^{35}$ and also with irradiation (data not shown). Nevertheless, in the present work, skin discrimination by speckle size is less efficient than by $S$. Hence, as observed in the previous work, ${ }^{35}$ speckle size seems to be less sensitive than $S$ to variations in optical properties of a scattering medium, and the evaluation of the mean dimension of the speckle grain by the fractal approach used seems more efficient for discrimination between irradiated and healthy skin. The Hurst coefficient characterizes intensity increments for the lowest neighborhood, i.e., into the speckle grain, and can be represented graphically by the slope of the diffusion curve at the origin [Eq. (6) and Fig. 2(b)]. Hence, a decrease in the Hurst coefficient $H$ reflects a decrease in the Hölderian regularity ${ }^{58}$ into the speckle grain. On the grain scale in a speckle pattern, an increase in Hölderian irregularity is observed after irradiation before an increase in the mean dimension of the grain $(S)$. Also, the increase in Hölderian irregularity is observed before clinical signs appear. An increase in the saturation of the variance $G$, and so in the speckle pattern contrast, is also observed before lesion emergence.

Explaining how the three model parameters vary with optical property changes caused by irradiation is a difficult task. First, because the changes in optical properties with irradiation are still unknown, and second, because more than one optical property change can take place simultaneously. Nevertheless, according to previous work, ${ }^{35}$ the first hypotheses to interpret parameter variations with irradiation can be formulated.

The different behaviors observed between these parameters with radiation, concerning their discrimination but also their variations, would mean that they are connected to different properties in skin. With the aim of trying to explain this, it is necessary to consider skin as a scattering medium composed of a complex mixture of different sizes of scatterers. In a previous study, we showed that the parameters $G, S$, and $H$ discriminated several media composed of monodispersepolystyrene microspheres of different sizes in solution, and mixtures of them. ${ }^{35}$ We pointed out that the Hurst coefficient and the self-similar element would characterize the scattering medium on the scatterer scale. They would be related to characteristics of scattering particles, and their variation with the changing scatterer size seemed to be caused by changes in angular distribution of backscattered light induced by the scatterer size change. ${ }^{35}$ Also, $H$ has a tendency to increase with microsphere size that is similar to the scattering efficiency factor. Inversely, $S$ has a tendency to decrease with scatterer size, which is similar to the anisotropy coefficient. ${ }^{35}$ Unlike parameters $H$ and $S$, the saturation of the variance $G$ seems not to be related to scatterer size, so it is difficult to interpret its significance in the medium. ${ }^{35}$ According to the variations observed in $H$ and $S$ with scatterer size ${ }^{35}$ and radiation, and according to their potential relationship with the medium on the scatterer scale, we can suppose that radiation has resulted in a decrease in size of scatterers in the skin. A concentration increase in small scatterers (Rayleigh scattering) in irradiated skin $(15 \mathrm{~Gy})$ was also proposed in an ex vivo study. ${ }^{68}$ Concerning the different discriminatory behaviors of these parameters, the results of the present study show that the self-similar element $S$ discriminates only when serious injuries have appeared, such as moist desquamation or necrosis (Table 2). Therefore, the Hurst coefficient $H$ seems to be more sensitive in detecting small changes in skin, i.e., on an invisible scale, whereas only large changes involve significant variation of $S$. Also in the previous study, ${ }^{35}$ small scatterers (Rayleigh scattering, diameter $0.2 \mu \mathrm{m}$ ) seem to influence the Hurst coefficient more than big scatterers (Mie scattering, diameter $2 \mu \mathrm{m})$, which influence the self-similarity parameter more (data not shown). Consequently, an increase in the proportion of small scatterers in skin with radiation dose, and the high sensitivity of $H$ to variations in small scatterers, can explain its potential for discrimination. Similarly, the low sensitivity of $S$ to variations in small scatterers can explain its inefficiency to discriminate irradiated from healthy skin. Currently, we are trying to explain the higher concentration of small scatterers in irradiated rather than in healthy skin by histological analysis of biopsied porcine samples, notably by collagen density measurements.

Further investigations are still required to understand what changes in optical properties of the skin might be associated with changes observed in speckle parameters. To find solutions for the inverse problem, numerical studies are needed on turbid media of known optical parameters (including scattering coefficient and anisotropy factor) and with known distribution in size and shape of scatterers, illuminated by coherent light with given illumination and detection conditions. Behaviors of speckle parameters could be thus compared according to any scattering change, e.g., changes in number, size, and distribution of scatterer size. In parallel, more studies on tissue phantoms could be carried out.

\section{Conclusions}

Characterization of the extent of radiation injury is essential to enable early optimal treatment for victims overexposed to ionizing radiation. In particular, for clinicians it is essential to estimate as soon as possible the extent of the irradiated cutaneous zone before clinical radio-induced signs appears, i.e., during the clinically latent postirradiation period. In this work, we present a noninvasive and innovative technique based on speckle phenomenon designed as a diagnostic aid.

Several speckle patterns are recorded in vivo from porcine skin at different times after radiation exposure. Three angles from specular reflection are used to acquire information from the surface to deep within the skin. A fractal speckle approach is used for speckle pattern processing. This approach allows us to extract three parameters from each speckle pattern that 
characterizes it on three different scales: the Hurst coefficient $H$, the self-similar element $S$, and the saturation of the variance $G$.

This noninvasive technique allows in vivo discrimination between irradiated and healthy skin in two minipigs of the Pitman-Moore strain locally irradiated with 40 Gy of radiation. This discrimination is possible during the clinically latent period, characteristic of radiation burns by two parameters: the Hurst coefficient $H$ and the saturation of the variance $G$. Discrimination by the Hurst coefficient is the most efficient. Discrimination by the self-similar element $S$ seems to be related to the appearance of serious clinical signs such as moist desquamation and necrosis. Discrimination becomes greater with time and is available for several observation angles from specular reflection that show that physiological modifications with radiation seem to occur at different depths in the skin. Different behaviors of speckle parameters with radiation show that they represent different changes in skin. In particular, the behaviors of $H$ and $S$ related to scatterer size seem to translate to a decrease in size of scatterers in skin with radiation. Currently, we are trying to explain this by histological analysis of biopsied porcine samples using collagen density measurements. The higher sensitivity of $H$ to variations in small scatterers in contrast to the $S$ parameter, combined with the increase in the proportion of small scatterers in irradiated skin, can explain the high potential of $H$ and the inefficiency of $S$ in discriminating irradiated from nonirradiated skin.

To complete skin exploration at different depths, the use of polarizers in different configurations might be useful, notably to improve discrimination. Moreover, radiation with other doses could be used to evaluate the sensitivity of the technique to detect radiation injury as a function of time after radiation exposure and before lesion emergence. Likewise, it is necessary to verify the potential of the technique with human skin, for example by testing it on patients treated with radiotherapy.

We wish to understand the optical significance of the speckle parameters used in this work by continuing to evaluate the relationship between their variations and changes in scattering media. Speckle study could become thus a noninvasive diagnostic aid, which would be of great interest to dermatologists.

\section{Acknowledgments}

The authors thank the Délégation Générale de l'Armement (DGA) for financial support. The authors would also like to thank D. Agay of Centre de Recherches du Service de Santé des Armées (CRSSA) for providing experimental support, and B. Clairac and G. Gruel (IRSN, Laboratoire de Dosimétrie Biologique) for their helpful scientific discussions.

\section{References}

1. A. V. Baranova, "Local radiation injury," Chap. 14 in Medical Management of Radiation Accident, 2nd ed., I. A. Gusev, A. K. Guskova, and F. A. Mettler, Eds., CRC Press, Boca Raton, FL (2001).

2. I. Clairand, F. Trompier, J. F. Bottolier-Depois, and P. Gourmelon, "Ex vivo ESR measurements associated with Monte Carlo calculations for accident dosimetry: application to the 2001 Georgian accident," Radiat. Prot. Dosim. 120, 500-505 (2006).

3. F. Trompier, et al., "EPR dosimetry for actual and suspected overexposures during radiotherapy treatments in Poland," Radiat. Meas. $\mathbf{4 2}$
1025-1028 (2007)

4. R. A. Reyes, A. Romanyukha, F. Trompier, C. A. Mitchell, I. Clairand, T. De, L. A. Benevides, and H. M. Swartz, "Electron paramagnetic resonance in human fingernails: the sponge model implication," Radiat. Environ. Biophys. 47, 515-526 (2008).

5. J. P. Pouget et al., "PCC-FISH in skin fibroblasts for local dose assessment: biodosimetric analysis of a victim of the Georgian radiological accident," Radiat. Res. 162, 365-376 (2004).

6. P. Gottlober, G. Krahn, and R. U. Peter, "The cutaneous radiation syndrome: clinics, diagnostics and therapy," Hautarzt 51, 567-574 (2000)

7. P. Gottlöber, G. Bezold, L. Weber, P. Gourmelon, J. M. Cosset, W. Bähren, H. J. Hald, T. M. Fliedner, and R. U. Peter, "The radiation accident in Georgia-Clinical appearance and diagnostics of cutaneous radiation syndrome," J. Am. Acad. Dermatol. 42, 453-458 (2000).

8. R. E. Goans, J. H. Cantrell, and F. B. Meyers, "Ultrasonic pulse echo determination of thermal injury in deep dermal burns," Med. Phys. 4(4), 259-263 (1977).

9. J. A. Brink, P. W. Sheets, K. A. Dines, M. R. Etchison, C. W. Hanke, and A. M. Sadove, "Quantitative assessment of burn injury in porcine skin with high-frequency ultrasonic imaging," Invest. Radiol. 21(8), 645-651 (1986).

10. J. A. Bauer and T. Sauer, "Cutaneous $10 \mathrm{MHz}$ ultrasound B scan allows the determination of burn depth," Burns Therm. Inj. 15(1), 49-51 (1989).

11. F. Daburon, Irradiation Aigues Localisées, Bases Radiobiologiques du Diagnostic et du Traitement, s.l, Edition de Physique, ISBN: 2-86883-313-6 (1997).

12. P. Gottlöber, M. Kerscher, H. C. Korting, and R. U. Peter, "Sonographic determination of cutaneous and subcutaneous fibrosis after accidental exposure to ionising radiation in the course of the Chernobyl nuclear power plant accident," Ultrasound Med. Biol. 23(1), 9-13 (1997).

13. R. E. Goans, R. H. Goans, R. E. Goans, Jr., and D. M. Christensen, "Ultrasonic analysis of acute thermal and radiation injury-a pilot study," Radiat. Meas. 42, 1261-1264 (2007).

14. S. Delanian and J. L. Lefaix, "Evaluation of late radiation-induced changes in superficial microcirculation. I. Clinical benefit of the cutaneous Doppler laser," Cancer Radiother 4, 408-414 (2000).

15. J. L. Lefaix and S. Delanian, "Evaluation of late radiation-induced changes in superficial microcirculation after acute beta-irradiation. II. Prognostic importance of cutaneous Doppler laser," Cancer Radiother 4, 415-420 (2000).

16. P. Lehmann, "Surface-roughness measurement based on the intensity correlation function of scattered light under speckle-pattern illumination," Appl. Opt. 38, 1144-1152 (1999).

17. P. Horvath, M. Hrabovsky, and P. Smid, "Application of speckle decorrelation method for small translation measurements," Opt. Appl. 34(2), 203-218 (2004).

18. O. Gilbert, C. Deumie, and C. Amra, "Angle-resolved ellipsometry of scattering patterns from arbitrary surfaces and bulks," Opt. Express 13(7), 2403-2418 (2005).

19. C. Hun, M. Bruynooghe, J. Caussignac, and P. Meyrueis, "Study of the exploitation of speckle techniques for pavement surface Characterization," Proc. SPIE 6341, 63412A (2006).

20. L. Brunel, A. Brun, and P. Snabre, "Microstructure movements study by dynamic speckle analysis," Proc. SPIE 6341, 634127 (2006).

21. D. A. Boas and A. G. Yodh, "Spatially varying dynamical properties of turbid media probed with diffusing temporal light correlation," $J$. Opt. Soc. Am. 14, 192-215 (1997).

22. I. V. Fedosov and V. V. Tuchin, "The use of dynamic speckle field space-time correlation function estimates for the direction and velocity determination of blood flow," Proc. SPIE 4434, 192-196 (2001).

23. J. D. Briers, G. Richard, and X. W. He, "Capillary blood flow monitoring using laser speckle contrast analysis (LASCA)," J. Biomed. Opt. 4(1), 164-175 (1999)

24. D. A. Zimnyakov, J. D. Briers, and V. V. Tuchin, "Speckle technologies for monitoring and imaging of tissues and tissue like phantoms," Chap. 18 in Handbook of Biomedical Diagnostics, V. V. Tuchin, Ed., SPIE Press, Bellingham, WA (2002).

25. J. F. Román, P. Fernández, V. Moreno, M. Abeleira, M. Gallas, and D. Suárez, "The mechanical behavior of human mandibles studied by electronic speckle pattern interferometry," Eur. J. Orthod. 21(4), 413-421 (1999).

26. D. Zhang, D. D. Arola, and J. A. Rouland, "Evaluating the elastic 
modulus of bone using electronic speckle pattern interferometry," Exp. Tech. 25, 32-34 (2001).

27. L. Yang, P. Zhang, S. Liu, P. R. Samala, M. Su, and H. Yokota, "Measurement of strain distributions in mouse femora with 3Ddigital speckle pattern interferometry," Opt. Lasers Eng. 45(8), 843851 (2007)

28. J. Zhang, G. C. Jin, L. B. Meng, L. H. Jian, A. Y. Wang, and S. B. Lu, "Strain and mechanical behaviour measurements of soft tissues with digital speckle method," J. Biomed. Opt. 10(3), 034021 (2005).

29. X. Yao, P. Wang, and R. Dai, "Experimental study od damage and fracture of cancellous bone using a digital speckle correlation method," J. Biomed. Opt. 13(3), 034026 (2008).

30. C. L. Benhamou, S. Poupon, E. Lespessailles, S. Loiseau, R. Jennane, V. Siroux, W. Ohley, and L. Pothuaud, "Fractal analysis of radiographic trabecular bone texture and bone mineral density: two complementary parameters related to osteoporotic fractures," J. Bone Miner. Res. 16(4), 697-704 (2001).

31. L. Pothuaud, E. Lespessailles, R. Harba, R. Jennane, V. Royant, E. Eynard, and C. L. Benhamou, "Fractal analysis of trabecular bone texture on radiographs: discriminant value in post menopausal osteoporosis," Osteoporosis Int. 8, 618-625 (1998).

32. G. M. Tosoni, A. G. Lurie, A. E. Cowan, and J. A. Burleson, "Pixel intensity and fractal analyses: detecting osteoporosis in perimenopausal and postmenopausal women by using digital panoramic images," Oral Surg. Oral Med. Oral Pathol. Oral Radiol. Endod. 102(2), 235-241 (2006).

33. T. Hyon Ha, U. Yoon, K. J. Lee, Y. W. Shin, J.-M. Lee, I. Y. Kim, K. S. Ha, S. I. Kim, and J. S. Kwon, "Fractal dimension of cerebral cortical surface in schizophrenia and obsessive-compulsive disorder," Neurosci. Lett. 384(1-2), 172-176 (2005).

34. A. P. Pentland, "Fractal-based description of natural scenes," IEEE Trans. Pattern Anal. Mach. Intell. 6(6), 661-674 (1984).

35. O. Carvalho, B. Clairac, M. Benderitter, and L. Roy, "Statistical speckle study to characterize scattering media: use of two complementary approaches," Opt. Express 15(21), 13817-13831 (2007)

36. J. W. Goodman, "Statistical properties of laser speckle pattern," in Laser Speckle and Related Phenomena, in the series "Topics in Applied Physics,” J. C. Dainty, Ed., Springer-Verlag, Berlin (1984).

37. L. I. Goldfisher, "Autocorrelation function and power spectral density of last-produced speckle pattern," J. Opt. Soc. Am. 55(3), 247-253 (1964).

38. J. W. Goodman, Speckle Phenomena in Optics: Theory and Applications, Roberts and Co. Publishers, Greenwood Village, NY (2007)

39. P. H. Van Cittert, "Die wahrscheinliche schwingungsverteilung in einer von einer lichtquelle direkt oder mittels einer linse beleuchteten ebene," Physica (The Hague) 1, 201-210 (1934).

40. F. Zernike, "The concept of degree of coherence and its application to optical problems," Physica (The Hague) 5, 785-795 (1938).

41. V. Rajan, B. Varghese, T. G. van Leeuween, and W. Steenbergen, "Speckle size and decorrelation time; space-time correlation analysis of coherent light dynamically scattered from turbid media," Opt. Сотmun. 281, 1755-1760 (2008).

42. R. Berlasso, F. Perez Quintian, M. A. Rebollo, C. A. Raffo, and N. G. Gaggioli, "Study of speckle size of light scattered from cylindrical rough surfaces," Appl. Opt. 39, 5811-5819 (2000)

43. Y. Piederrière, J. Le Meur, J. Cariou, J. Abgrall, and M. Blouch, "Particle aggregation monitoring by speckle size measurement; application to blood platelets aggregation," Opt. Express 12, 4596-4601 (2004).

44. Y. Piederrière, J. Cariou, Y. Guern, B. Le Jeune, G. Le Brun, and J. Lortrian, "Scattering through fluids: speckle size measurement and Monte Carlo simulations close to and into the multiple scattering," Opt. Express 12, 176-188 (2004).

45. Y. Piederrière, F. Boulvert, J. Cariou, B. Le Jeune, Y. Guern, and G. Le Brun, "Backscattered speckle size as a function of polarization: influence of particle-size and- concentration," Opt. Express 13, 50305039 (2005).

46. J. A. Lock, "Role of multiple scattering in cross-correlated light scattering with a single laser beam," Appl. Opt. 36(30), 7559-7570 (1997).

47. P. Zakharov, S. Bhat, P. Schurtenberger, and F. Scheffold, "Multiplescattering suppression in dynamic light scattering based on a digital camera detection scheme," Appl. Opt. 45, 1756-1764 (2006).

48. J. M. Horta and V. M. Castaño, "Speckle interferometry from fiberreinforced materials: a fractal geometry approach," Optik (Stuttgart) 112(1), 21-25 (2001).

49. K. Uno, J. Uozumi, and T. Asakura, "Speckle clustering in diffraction patterns of random objects under ring-slit illumination," Opt. Commun. 114, 203-210 (1995).

50. K. Uno, J. Uozumi, and T. Asakura, "Correlation properties of speckles produced by diffractal-illuminated diffusers," Opt. Commun. 124, 16-22 (1996).

51. J. Uozumi, M. Ibrahim, and T. Asakura, "Fractal speckles," Opt. Commun. 156, 350-358 (1998).

52. H. Funamizu and J. Uozumi, "Generation of fractal speckles by means of a spatial light modulator," Opt. Express 15, 7415-7422 (2007).

53. J. Horta, W. Brostow, G. Martinez, and V. M. Castaño, "Characterization of bones by speckle interferometry," J. Med. Eng. Technol. 27(2), 49-53 (2003).

54. Z. Li, H. Li, and Y. Qiu, "Fractal analysis of laser speckle for measuring roughness," Proc. SPIE 6027, 60271S (2006).

55. D. A. Zimnyakov, V. V. Tuchin, and A. A. Mishin, "Spatial speckle correlometry in applications to tissue structure monitoring," Appl. Opt. 36, 5594-5607 (1997).

56. S. Guyot, M. C. Péron, and E. Deléchelle, "Spatial speckle characterization by Brownian motion analysis," Phys. Rev. E 70, 046618 (2004).

57. P. Abry, P. Gonçalves, and P. Flandrin, Wavelets, Spectrum Analysis and $1 /$ f Processes, Springer, Berlin (1995).

58. M. F. Barnsley, R. L. Devaney, B. B. Mandelbrot, H. O. Peitgen, D. Saupe, and R. F. Voss, The Science of Fractal Images, Springer, New York (1988).

59. T. D. Frank, A. Daffertshofer, and P. J. Beek, "Multivariate OrnsteinUhlenberg processes with mean field-dependent coefficientsapplication to postural sway," Phys. Rev. E 63, 011905 (2000).

60. G. A. Losa and T. F. Nonnenmacher, "Self-Similarity and fractal irregularity in pathologic tissues," Mod. Pathol. 9(3), 174-182 (1996).

61. S. S. Cross, "Fractals in pathology," J. Pathol. 182, 1-8 (1997).

62. G. G. Losa, "The fractal geometry of life," Riv. Biol. 102(1), 29-59 (2009).

63. Q. B. Li and F. P. Chiang, "Three-dimensional of laser speckle," Appl. Opt. 31, 6287-6291 (1992).

64. T. L. Alexander, J. E. Harvey, and A. R. Weeks, "Average speckle size as a function of intensity threshold level: comparison of experimental measurements with theory," Appl. Opt. 33, 8240-8250 (1994).

65. R. V. Hogg and J. Ledolter, Engineering Statistics, Macmillan Publishing Co., New York (1987).

66. B. Escofier and J. Pagès, Analyses Factorielles Simples et Multiples: Objectifs, Méthodes et Interprétation, Dunod Eds., Paris (1998).

67. S. P. Morgan and M. E. Ridgway, "Polarization properties of light backscattered from a two layer scattering medium," Opt. Express 7, 395-402 (2000).

68. F. Boulvert, "Analyse de milieux fortement diffusants par polarimétrie de Mueller et méthodes optiques cohérentes. Application à l'étude du syndrome cutané d'irradiation aiguë," Thèse de Doctorat, UBO (2006). 